\title{
Status of the NICA Project
}

\section{V.D. Kekelidze, A.D. Kovalenko, A.M.Korotkova ${ }^{1}$, R.I. Lednicky, V.A.Matveev, I.N. Meshkov, A.S. Sorin, G.V. Trubnikov (for the NICA Collaboration)}

Joint Institute for Nuclear Research

Joliot Curie, 6, Dubna, Russia, 141980

E-mail: Kekelidze@jinr.ru

\begin{abstract}
Nuclotron-based Ion Collider facility (NICA) project is under realization in the Joint Institute for Nuclear Research (JINR) in Dubna. The main goal of the project is the studying of hot and dense strongly interacting matter in heavy ion collisions with the MultiPurpose Detector (MPD). At the NICA collider will provide ion beam collisions at the center of mass energy $\sqrt{ } \mathrm{s}_{\mathrm{NN}}=4-11$ $\mathrm{GeV}$ (NN-equivalent) at average luminosity of $10^{27} \mathrm{~cm}^{-2} \mathrm{~s}^{-1}$ for $\mathrm{Au}(79+)$ and in the experiment Baryonic Matters at Nuclotron (BM@N) at the extracted beam from the upgraded superconducting synchrotron Nuclotron with energies up to $5.81 \mathrm{GeV} / \mathrm{u}$ (scaled to $\mathrm{A} / \mathrm{Z}=2$ ). The program also foresees the further spin physics research with colliding beams of polarized deuterons and protons at the energies up to $\sqrt{ } \mathrm{s}=26 \mathrm{GeV}$ and $\sqrt{\mathrm{s}_{\mathrm{NN}}}=13 \mathrm{GeV}$ for protons and deuterons respectively. General design and construction status of the complex is presented, whereas some fresh results from current R\&D program are briefly introduced.
\end{abstract}

The European Physical Society Conference on High Energy Physics

18-24 July, 2013

Stockholm, Sweden

\footnotetext{
${ }^{1}$ Speaker
} 


\section{Introduction}

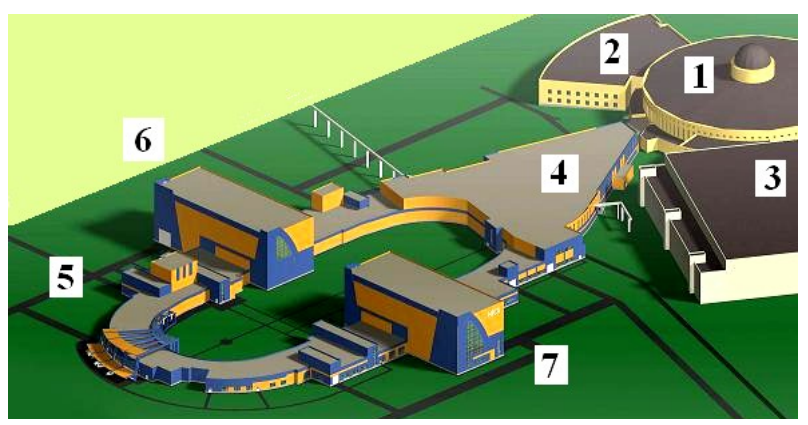

Figure 1: the NICA facility architectural view: $1,2,3$ the existing buildings of the Nuclotron complex, 4,5,6,7 - new buildings and objects, namely: 4,5 - NICA collider with beam injection lines, 6 - the MPD building, 7 - the future SPD building. Perimeter of the collider is of $503 \mathrm{~m}$.

The Nuclotron-based Ion Collider fAcility (NICA) in Dubna [1,2] will provide a major infrastructure for forefront research in a globally competitive field of particle physics: the exploration of extreme states of matter including phase transitions between them; and investigation of nucleon spin structure and polarization phenomena. The general view of the NICA is shown at the Figure 1.

Of particular interest is the hadron-to-quark matter transition with its main aspects: chiral symmetry restoration, deconfinement and color superconductivity. To perform precision studies with a discovery potential in this field, dedicated heavy-ion collision experiments with fine steps scanning on the energy and system size, with acceptance coverage close to four-pi geometry, and providing high performance in particle identification and energy resolution, are required. The Multi-Purpose Detector (MPD) allowing to accumulate high statistics data. The fixed target experiment Baryonic Matter at Nuclotron (BM@N) is under preparation at the extracted beams (energy range from 1.5 to $5.81 \mathrm{GeV} / \mathrm{u}$ for $\mathrm{A} / \mathrm{Z}=2$ ). Commissioning of the setups MPD and BM@N should be started in 2017 and 2015 respectively.

\section{NICA-MPD Physics plan (stage 1)}

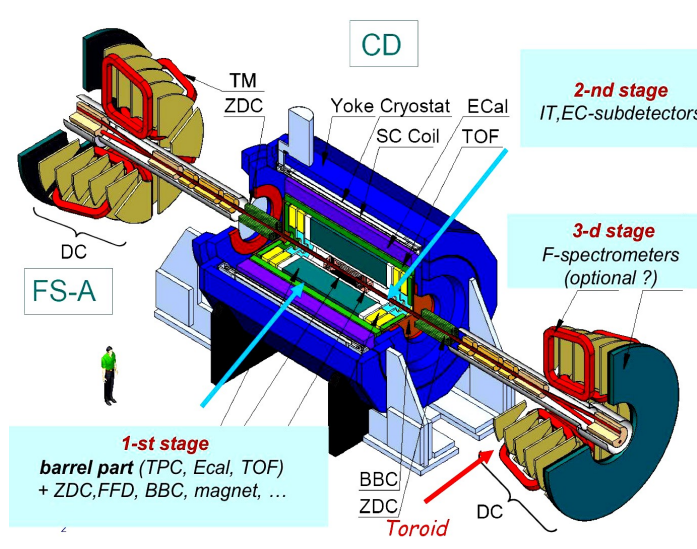

Figure 2: General view of the MPD
As the first physics measurements: an energy-system size scan will be performed at NICA-MPD with the chosen beam species varying the collisions energy from 4 to $11 \mathrm{GeV}$ in steps of 1-2 GeV. Measurements of hadrons (p, K, (anti)p, (anti)hyperons, light (anti)nuclei and dilepton spectra as a function of energy, system size, centrality, $\mathrm{p}_{\mathrm{T}}$, rapidity and azimuthal angle. The search strategy: 1) scanning the chosen energy interval trying to find the peculiarities associated with the QCD critical end point (CEP); 2) detailed study of the LMR dilepton enhancement in the 


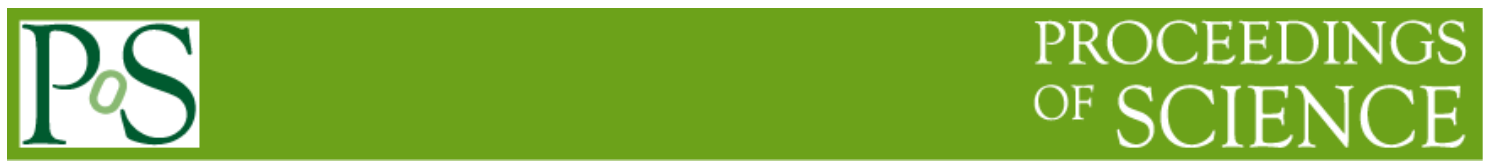

unexplored region of the highest baryon density. If an indication for dropping mass found à detailed look in this region. The scheme of the MPD is presented at the Figure 2. Basic characteristics of the MPD: SC Magnet: 0.5 T; Tracking: TPC; Particle ID: TOF, ECAL, TPC; T0, Triggering: FFD; Centrality, Event plane: ZDC. MPD advantages: 1) hermeticity, homogenous acceptance ( $2 \pi$ in azimuth), low material budjet; 2) good tracking performance and powerful PID (hadrons, e,ץ); 3) high event rate capability and careful event characterization.

\section{Recent $R \& D$ results}

The progress achieved in the accelerator complex was presented at the IPAC'2013 [3]. As the most important result for NICA collider, the experimental study of a beam stochastic cooling system model at the Nuclotron circulating beam can be mentioned. The development of NICA injection complex is actively performed. New ESIS heavy ions source KRION-6T with solenoid of 6 Tesla is assembled and is under commissioning. New source of polarized particles (SPI) had been assembled and at the test bench now. New $3 \mathrm{MeV} / \mathrm{u}$ heavy-ion RFQ linear accelerator is manufactured by BEVATECH Company (Frankfurt, Germany). The new heavy ion linac commissioning at Dubna is scheduled for 2014.

Prototypes of the main MPD parts such as Electromagnetic CALorimeter (ECAL), Time-Projection Chamber (TPC), Fast Forward Detector (FFD), Time of Flight stystem (TOF) have been made and they are being tested now.

Experimental area for BM@N experiment under construction now and results of the first extraction of the $3.42 \mathrm{~A} \mathrm{GeV}{ }^{12} \mathrm{C}$ beam at Nuclotron and its transportation to the setup is presented [4].

The NICA facility design and construction is in progress. The realization schedule foresees staging of the accelerator facility manufacturing/assembling and the MPD components as well. The collider technical project have been officially sent for Russian state expertize and approved with some conditions. We are planning to start preparatory work for the collider infrastructure build in this year.

\section{References}

[1] V.Kekelidze et al, in proceedings of ICHEP'2012, Melburn, Australia,

[2] NICA at JINR: http://nica.jinr.ru

[3] N.Agapov et al. NICA Project at JINR, in: proceedings of IPAC’2013, Shanghai, China, 2013.

[4] Physics of Particles and Nuclei Letters, 2012, Vol.9, No.8, 589-592. 